\title{
DINÂMICA NICTIMERAL E VERTICAL DAS CARACTERÍSTICAS LIMNOLÓGICAS EM AMBIENTE DE CRIAÇÃO DE PEIXES EM TANQUES-REDE
}

\author{
Odair Diemer, ${ }^{1}$ Dacley Hertes Neu, ${ }^{2}$ Aldi Feiden, ${ }^{3}$ Evandro Kleber Lorenz, ${ }^{4}$ \\ FÁbio Bittencourt ${ }^{5}$ e Wilson Rogerio Boscolo ${ }^{6}$ \\ 1. Mestrando em Zootecnia na área de nutrição e alimentação animal, UNIOESTE. E-mail: odairdiemer@hotmail.com \\ 2. Mestrando em Recursos Pesqueiros e Engenharia de Pesca, Unioeste, campus Toledo-PR \\ 3. Professor adjunto da Universidade Estadual do Oeste do Paraná \\ 4. Mestre em Zootecnia, Técnico do GEMAq - Grupo de Estudos em Manejo na Aquicultura \\ 5. Doutorando em Aquicultura pelo CAUNESP, Jaboticabal, SP \\ 6. Professor adjunto da Universidade Estadual do Oeste do Paraná.
}

\section{RESUMO}

O presente trabalho teve como objetivo verificar a dinâmica nictimeral e vertical das características limnológicas em ambiente de criação de peixes nativos em tanques-rede no reservatório da Itaipu Binacional. Os parâmetros avaliados foram temperatura da água, oxigênio dissolvido, condutividade elétrica, $\mathrm{pH}$, fósforo total, nitrito e amônia. Verificou-se que ocorreu variação nictimeral para todos os parâmetros, exceto para amônia e fósforo total.
Entretanto, as variáveis estão dentro dos limites recomendados para a aquicultura, com exceção do oxigênio dissolvido, que apresentou valores críticos à noite. Para a distribuição vertical, as concentrações dos parâmetros físicos e químicos da água não ultrapassaram o limite estabelecido pela resolução do CONAMA $357 / 05$ para criação de peixes. No entanto, houve variação vertical para nitrito e fósforo.

PALAVRAS-CHAVES: Aquicultura, cultivo intensivo, limnologia, qualidade de água.

\section{ABSTRACT}

\section{DIEL AND VERTICAL DYNAMIC OF LIMNOLOGICAL CHARACTERISTICS IN FISH REARING NET-CAGES ENVIROMENT}

This study aimed to verify the diel and vertical dynamic characteristics in limnological environment for rearing native fish in net-cage at the reservoir of Itaipu Binacional. The parameters evaluated were water temperature, dissolved oxygen, electrical conductivity, $\mathrm{pH}$, phosphorus, nitrite and ammonia. It was found that there was diel variation for all parameters, except for ammonia and phosphorus. But the variables are in the recommended limits for aquaculture, with the exception of dissolved oxygen that showed critical rates at night. For the vertical distribution concentrations of physical and chemical parameters of water did not exceed the limit established by CONAMA Resolution 357/05 for fish rearing, however, there was vertical variation for nitrite and phosphorus

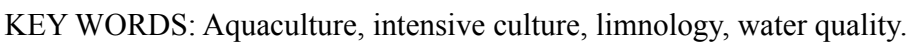

\section{INTRODUÇÃO}

O Brasil tem potencial pesqueiro como poucos países do mundo, pela quantidade de águas marítimas e continentais. Entretanto, somente $1 \%$ dos corpos d'água provenientes de barramento, lagos, lagoas, açudes, depósitos de águas pluviais e remansos de rios é liberado para produção de pescado (OSTRENSKY 
et al., 2008). O aproveitamento dos recursos hídricos existentes, principalmente dos reservatórios de hidrelétricas, tem proporcionado o desenvolvimento da criação de peixes em tanques-rede.

Por volta dos anos 1980 (AYROZA et al., 2006), iniciou-se o cultivo de peixes em tanques-rede, uma das formas mais intensivas de criação de peixes. Nesse sistema, há a otimização do processo de produção, podendo-se conseguir uma elevada produtividade em um determinado espaço, bem como incrementar consideravelmente a produção aquícola, além de diminuir a pressão sobre os estoques pesqueiros naturais, o que requer, entretanto, monitoramento regular das condições ambientais.

Trata-se de sistema de produção que é uma alternativa de investimento de menor custo e maior rapidez de implantação, sendo apontada como um agronegócio capaz de melhorar as condições sociais e econômicas de uma região.

Contudo, o desenvolvimento desse tipo de atividade produtiva apresenta riscos, por deteriorar a qualidade da água, quando não realizada de forma sustentável. Disso resultam prejuízos financeiros, afetando o crescimento, a reprodução, a saúde e a sobrevivência dos peixes.

Os ambientes aquáticos são dinâmicos e podem sofrer grandes variações nas suas características físicas e químicas ao longo tempo. As variações espaciais podem causar heterogeneidade significativa na distribuição de nutrientes (HAKANSON et al., 2000), pois, em corpos d'água, onde o material orgânico é predominantemente autóctone, o pool de nutrientes do epilímnio depende do equilíbrio entre as perdas por sedimentação e aumento dos fluxos de nutrientes do hipolímnio. Dessa forma, mudanças no período de 24 horas (nictimeral) podem ser maiores do que alterações que ocorrem em um ciclo anual. Assim, estudos sobre a variação nictimeral são de suma importância para a compreensão da dinâmica de um ecossistema.

Em locais lênticos, como reservatórios, é comum a ocorrência de estratificação térmica e química produzindo distribuição heterogênea dos compostos físicos e químicos ao longo da coluna d'água. Como gases nocivos e minerais podem ser liberados dos sedimentos (GUNKEL, 2003) que, com a circulação da água, misturam-se por entre a massa de água, há o risco de ocorrer mortandade de peixes. Por isso, o conhecimento do perfil vertical dos parâmetros físicos e químicos é fundamental.

O presente trabalho teve como objetivo verificar a dinâmica nictimeral e vertical das características limnológicas em ambiente de criação de peixes nativos em tanques-rede no reservatório da Itaipu Binacional, localizado no Refúgio Biológico do município de Santa Helena, PR.

\section{MATERIAL E MÉTODOS}

O estudo foi realizado no Centro de Desenvolvimento de Tecnologias para Piscicultura em Tanquesrede, localizado no Refúgio Biológico do município de Santa Helena, constituído por setenta tanques-rede de pequeno volume $\left(5 \mathrm{~m}^{3}\right)$ com dimensões de $2,0 \times 2,0 \mathrm{x}$ 1,5 metros de comprimento, largura e profundidade, respectivamente, destinados à produção de espécies nativas como o pacu (Piaractus mesopotamicus), a piracanjuba (Brycon orbignyanus) a piapara (Leporinus obtusidens) dentre outros.

Realizou-se a coleta da água nos dias 20 e 21 de março de 2009. Para verificar a dinâmica nictimeral, foram feitas amostragens durante um período de vinte e quatro horas, com um intervalo de três horas entre os procedimentos, totalizando oito horários diferentes, sendo às 10:00, 13:00, 16:00, 19:00, 22:00, 01:00, 04:00 e 07:00 horas. Para o levantamento dos dados abióticos, estabeleceram-se quatro estações de amostragem, de modo que toda a área dos tanquesrede fosse abrangida. Cada local foi considerado como uma repetição. As estações foram assim denominadas: 1S e 2S, localizadas nas coordenadas geográficas

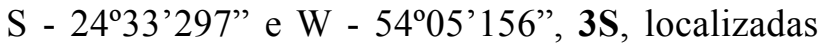

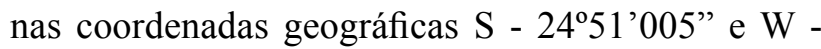
$54^{\circ} 21^{\prime} 446^{\prime \prime}$ e 4S, localizada nas coordenadas geográficas S - 24'51'004" e W - 54'21'449".

Para ser determinada a dinâmica vertical, coletaram-se amostras com auxílio de uma garrafa de Van Dorn em três horários diferentes - às 10:00, 13:00 e 16:00 horas -, sendo obtidas em três profundidades: E - epilímnio (camada superficial - $0 \mathrm{~m}$ de profundidade), M - metalímnio (camada intermediária $-6 \mathrm{~m}$ de profundidade) e $\mathrm{H}$ - hipolímnio (camada profunda $-12 \mathrm{~m}$ de profundidade).

A temperatura da água, o oxigênio dissolvido, a condutividade elétrica e o $\mathrm{pH}$ foram medidos in situ, 
isto é, no local, mediante potenciômetros portáteis Hanna Instruments ${ }^{\circledR}$. Preservaram-se as parcelas de água coletadas em garrafas de polietileno, sendo conservadas resfriadas para posterior análise. No Laboratório de Controle de Qualidade do Grupo de Estudos em Manejo na Aquicultura GEMAq/UNIOESTE, localizado na cidade de Toledo, PR, determinaram-se os parâmetros fósforo, nitrito e amônia, seguindo a metodologia proposta por MACKRETH et al. (1978), STRICKLAND \& PARSON (1972) e KOROLEFF et al. (1976), respectivamente.

Os dados foram submetidos à análise de variância ANOVA (ZAR, 1984) e quando observadas diferenças significativas $(\mathrm{p}<0,05)$, aplicou-se o teste de Tukey, em 5\% de significância, utilizando-se o programa SAS (2004).

\section{RESULTADOS E DISCUSSÃO}

Os valores dos parâmetros abióticos estão apresentados na Tabela 1. Observou-se que a dinâmica nictimeral dos parâmetros coletados in situ apresentaram diferença estatística $(\mathrm{p}<0,05)$ entre os diferentes horários de coleta (Tabela 1).

TABELA 1. Valores dos parâmetros abióticos no ambiente de criação de peixes em tanques-rede, coletados in situ

\begin{tabular}{lcccc}
\hline \multicolumn{5}{c}{ Parâmetros } \\
\hline Horários & $\mathrm{pH}$ & Temperatura da água $\left({ }^{\circ} \mathrm{C}\right)$ & Oxigênio $(\mathrm{mg} / \mathrm{L})$ & Condutividade $(\mu \mathrm{S} / \mathrm{cm})$ \\
\hline $10: 00$ & $7,59 \pm 0,09 \mathrm{a}$ & $29,67 \pm 0,09 \mathrm{~b}$ & $6,65 \pm 0,31 \mathrm{a}$ & $45 \pm 2,10 \mathrm{a}$ \\
$13: 00$ & $7,45 \pm 0,04 \mathrm{a}$ & $30,42 \pm 0,09 \mathrm{a}$ & $5,7 \pm 0,39 \mathrm{~b}$ & $46,97 \pm 2,42 \mathrm{a}$ \\
$16: 00$ & $7,17 \pm 0,04 \mathrm{dc}$ & $30,72 \pm 0,12 \mathrm{a}$ & $6,12 \pm 0,09 \mathrm{~b}$ & $41,12 \pm 0,15 \mathrm{~b}$ \\
$19: 00$ & $7,1 \pm 0,04 \mathrm{~d}$ & $30,37 \pm 0,12 \mathrm{a}$ & $4,62 \pm 0,58 \mathrm{bc}$ & $40,07 \pm 0,48 \mathrm{~b}$ \\
$22: 00$ & $6,7 \pm 0,17 \mathrm{e}$ & $29,87 \pm 0,26 \mathrm{~b}$ & $3,7 \pm 0,75 \mathrm{bc}$ & $38,06 \pm 1,72 \mathrm{~b}$ \\
$01: 00$ & $7,09 \pm 0,10 \mathrm{~d}$ & $29,82 \pm 0,22 \mathrm{~b}$ & $3,7 \pm 0,75 \mathrm{bc}$ & $38,6 \pm 1,72 \mathrm{~b}$ \\
$04: 00$ & $7,26 \pm 0,03 \mathrm{bcd}$ & $29,67 \pm 0,10 \mathrm{~b}$ & $3,02 \pm 0,26 \mathrm{dc}$ & $39,62 \pm 0,21 \mathrm{~b}$ \\
$07: 00$ & $7,36 \pm 0,02 \mathrm{abc}$ & $29,2 \pm 0,25 \mathrm{c}$ & $3,12 \pm 0,17 \mathrm{~d}$ & $40,15 \pm 0,52 \mathrm{~b}$ \\
C.V. $(\%)$ & 1,19 & 0,58 & 10,44 & 3,51 \\
F (p) & 36,9 & 32,54 & 34,93 & 17,92 \\
\hline
\end{tabular}

Valores na mesma coluna seguidos da mesma letra não diferem estatisticamente $(\mathrm{P}>0,05)$ pelo teste de Tukey.

A temperatura da água apresentou variação de 28,9 a $30,8^{\circ} \mathrm{C}$ no período de vinte quatro horas. Para espécies tropicais, a faixa adequada de temperatura geralmente varia de 28 a $32{ }^{\circ} \mathrm{C}$. Portando, mantevese dentro dos limites para a criação de peixes (KUBITZA, 1999). Verificou-se que o $\mathrm{pH}$ demonstrou variação nictimeral de 6,5 a 7,6, encontrando-se dentro da faixa tolerável para criação de peixes nativos. Segundo KUBITZA (1999), a escala adequada de $\mathrm{pH}$ para a produção de peixes está compreendida entre 6,5 a 9,0, sendo que valores acima ou abaixo podem prejudicar o desenvolvimento dos animais. Esse parâmetro pode variar durante o dia, em função da atividade fotossintética e respiratória das comunidades aquáticas.
Após as 22 horas, o oxigênio da água mostrou números inferiores a $4 \mathrm{mg} \mathrm{L}^{-1}$. Entretanto, não se verificaram níveis menores do que $2,76 \mathrm{mg} \mathrm{L}^{-1}$. O mesmo ciclo de oxigênio, apresentando baixas quantidades à noite, foi observado por SILVA \& MENDES (2006) em cultivo de pós-larvas de camarão marinho. Segundo ZIMERMANN et al. (2001), concentrações entre 2,0 e 4,0 $\mathrm{mg} \mathrm{L}^{-1}$ podem provocar um crescimento lento aos organismos quando expostos continuamente. A concentração desse gás na água varia com a temperatura, bem como depende ainda da pressão atmosférica e da salinidade da água. Em lagos, ocorre variação contínua do oxigênio durante o dia, em consequência de processos físicos, químicos e biológicos, podendo apresentar teores críticos no período noturno, como o verificado neste trabalho. 
GRAEFF \& PRUNER (2006), estudando as variáveis que podem interferir na criação de tilápias em viveiros, relatam que a estratificação térmica e a contínua aeração formada pela corrente da água com ajuda dos ventos contribuem para que não ocorra a diminuição de oxigênio e colabore para a não ocorrência de amônia em sistemas de cultivo. Isso também foi relatado por LIMA et al. (2008), que não observaram alterações negativas na qualidade da água do canal de irrigação com criação de peixes. Contudo, os autores relatam que a velocidade de vazão e o bombeamento da água foram decisivos para um cultivo produtivo nesse sistema. SANTOS et al. (2009), que criaram tilápias em esgoto doméstico tratado, também relatam a eficiência da aeração mecânica no cultivo de alevinos de tilápias.

Cada organismo apresenta um limite ideal de oxigênio dissolvido na água para sua sobrevivência. Contudo, viveiros que contêm valores acima de $4 \mathrm{mg} \mathrm{L}^{-1}$ apresentam boas condições para criação de peixes (ZIMERMANN et. al., 2001). Assim, teores mais baixos podem levar ao estresse dos animais.

Segundo ARANA (2004), o nível de oxigênio dissolvido durante o dia pode aumentar em virtude dos processos fotossintéticos. No entanto, durante a noite, a respiração biológica e a deterioração química do sedimento provocam perda desse elemento, que pode alcançar níveis críticos, causando sérios riscos aos animais cultivados. Dessa maneira, justifica-se a queda de oxigênio ocorrido durante o período noturno.

Os resultados deste trabalho estão próximos aos encontrados por CHAGAS et al. (2007), que estudaram a produtividade do tambaqui em tanques-rede no lago de Ariauzinho (AM). Os autores relatam que o perfil do oxigênio variou de 2,59 a $5,72 \mathrm{mg} / \mathrm{L}^{-1}$, e a amônia e o pH mantiveram-se estáveis. Entretanto, os resultados aumentaram no último mês de cultivo, o que pode ter sido provocado por entrada de água de um afluente do reservatório.

SALVADOR et al. (2003) relatam que os parâmetros limnológicos do cultivo intensivo de tilápias se mantêm dentro da faixa imposta pela legislação. Isso também foi exposto por CARNEIRO et al. (1999), que não observaram deterioração na qualidade de água no cultivo de tilápia-vermelha em tanques-rede, e relatam, ainda, que o crescimento dos peixes não foi afetado pelo aerador que proveu movimentação de água.

No presente trabalho, a condutividade elétrica

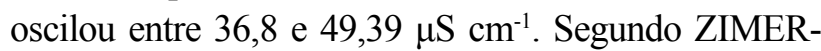
MANN (2001), os valores desejáveis para a criação de peixes encontram-se entre 20 a $150 \mu \mathrm{S} \mathrm{cm}^{-1}$. Portanto, os resultados obtidos no presente estudo estão dentro da faixa confortável. Esse fator ajuda a detectar fontes poluidoras no sistema e cresce linearmente com a quantidade da concentração de sais no meio aquático (RIBEIRO et al., 2005). Quando seus valores são altos, indicam grau de decomposição elevado e o inverso (valores reduzidos) indica acentuada produção primária, sendo, portanto, uma maneira que possibilita avaliar a disponibilidade de nutrientes nos ecossistemas aquáticos (ESTEVES, 1998).

Nos diferentes horários de coleta, somente o nitrito apresentou diferença estatística $(\mathrm{P}<0,05)$ entre as variáveis analisadas (Tabela 2).

TABELA 2. Valores dos parâmetros abióticos do ambiente de criação de peixes em tanques-rede, analisados em laboratório

\begin{tabular}{lccc}
\hline \multicolumn{3}{c}{ Parâmetros } \\
\hline Horários & Nitrito $\mu \mathrm{g} / \mathrm{L}$ & Amônia $\mu \mathrm{g} / \mathrm{L}$ & Fósforo Total $\mu \mathrm{g} / \mathrm{L}$ \\
\hline $10: 00$ & $1,57 \pm 0,54 \mathrm{dc}$ & $28,77 \pm 6,95 \mathrm{a}$ & $40,12 \pm 18,52 \mathrm{a}$ \\
$13: 00$ & $2,44 \pm 0,67 \mathrm{bc}$ & $12,2 \pm 2,75 \mathrm{a}$ & $28,87 \pm 14,91 \mathrm{a}$ \\
$16: 00$ & $4,02 \pm 0,77 \mathrm{ab}$ & $42,2 \pm 24,92 \mathrm{a}$ & $25,75 \pm 7,07 \mathrm{a}$ \\
$19: 00$ & $4,23 \pm 0,83 \mathrm{a}$ & $29,08 \pm 19,91 \mathrm{a}$ & $28,25 \pm 6,12 \mathrm{a}$ \\
$22: 00$ & $1,3 \pm 0,21 \mathrm{~d}$ & $19,7 \pm 14,21 \mathrm{a}$ & $22 \pm 6,61 \mathrm{a}$ \\
$01: 00$ & $1,52 \pm 0,12 \mathrm{~cd}$ & $18,45 \pm 12,38 \mathrm{a}$ & $32 \pm 8,77 \mathrm{a}$ \\
$04: 00$ & $2,12 \pm 0,11 \mathrm{~cd}$ & $4,89 \pm 3,50 \mathrm{a}$ & $27 \pm 1,44 \mathrm{a}$ \\
$07: 00$ & $1,41 \pm 0,46 \mathrm{~d}$ & $15 \pm 10,81 \mathrm{a}$ & $46,37 \pm 36,36 \mathrm{a}$ \\
C.V. $(\%)$ & 30,6 & 33,94 & 12,19 \\
F (p) & 18,95 & 2,8 & 1,19 \\
\hline
\end{tabular}

Valores na mesma coluna seguidos da mesma letra não diferem estatisticamente $(\mathrm{P}>0,05)$ pelo teste de Tukey. 
Observou-se que o nitrito, a amônia e o fósforo total apresentaram valores variando de 1,18 a 5,06 $\mu \mathrm{g} \mathrm{L}{ }^{-1}, 1,39$ a $48,99 \mu \mathrm{g} \mathrm{L}^{-1}$ e 15,39 a $82,73 \mu \mathrm{g} \mathrm{L}^{-1}$, respectivamente. Essas concentrações observadas não prejudicam a criação de organismos aquáticos, segundo os limites citados por KUBITZA (1999), e não ultrapassam o estabelecido pela Resolução 357/05 do CONAMA, para águas de classe II.
Na Tabela 3, verifica-se a dinâmica vertical das características limnológicas. Apenas o nitrito, a amônia e o fósforo total foram significativamente diferentes em nível de 5\%. A temperatura e o oxigênio dissolvido da água mantiveram valores próximos em todos os locais de coleta, não apresentando oscilações significativas ( $>0,05)$, o que pode estar relacionado com a circulação de água nesse braço do reservatório.

TABELA 3. Parâmetros físicos e químicos da água do cultivo em diferentes estratos

\begin{tabular}{lccccc}
\hline Variáveis & Epilímnio & Metalímnio & Hipolímnio & C.V. $(\%)$ & $\mathrm{F}(\mathrm{p})$ \\
\hline $\mathrm{pH}$ & $7,39 \pm 0,14 \mathrm{a}$ & $7,49 \pm 0,16 \mathrm{a}$ & $7,35 \pm 0,13 \mathrm{a}$ & 4,34 & 1,11 \\
Temperatura da água $\left({ }^{\circ} \mathrm{C}\right)$ & $30,27 \pm 0,48 \mathrm{a}$ & $29,87 \pm 0,36 \mathrm{a}$ & $29,86 \pm 0,28 \mathrm{a}$ & 3,14 & 1,47 \\
Oxigênio $\left(\mathrm{mg} \mathrm{L}^{-1}\right)$ & $5,57 \pm 0,49 \mathrm{a}$ & $5,23 \pm 0,77 \mathrm{a}$ & $5,21 \pm 0,76 \mathrm{a}$ & 12,55 & 0,27 \\
Condutividade $\left(\mu \mathrm{S} \mathrm{cm}^{-1}\right)$ & $44,23 \pm 2,81 \mathrm{a}$ & $46,35 \pm 4,54 \mathrm{a}$ & $45,20 \pm 5,68 \mathrm{a}$ & 8,62 & 0,22 \\
Nitrito $\left(\mu \mathrm{g} \mathrm{L}^{-1}\right)$ & $2,71 \pm 1,41 \mathrm{~b}$ & $2,71 \pm 1,81 \mathrm{~b}$ & $12,74 \pm 5,54 \mathrm{a}$ & 49,56 & 11,14 \\
Amônia $\left(\mu \mathrm{g} \mathrm{L}^{-1}\right)$ & $35,33 \pm 6,85 \mathrm{~b}$ & $43,15 \pm 22,10 \mathrm{ab}$ & $103,11 \pm 34,67 \mathrm{a}$ & 39,75 & 9,49 \\
Fósforo total $\left(\mu \mathrm{g} \mathrm{L}^{-1}\right)$ & $25,75 \pm 5,20 \mathrm{~b}$ & $38,52 \pm 4,11 \mathrm{~b}$ & $216,02 \pm 68,63 \mathrm{a}$ & 36,9 & 28,54 \\
\hline
\end{tabular}

Valores na mesma linha seguidos da mesma letra não diferem estatisticamente $(\mathrm{P}>0,05)$ pelo teste de Tukey.

$\mathrm{O} \mathrm{pH}$ variou pouco, não resultando em diferenças significativas $(\mathrm{p}>0,05)$ para os estratos. Esses valores encontrados estão dentro da faixa ótima para criação de peixes, caracterizando a água como neutra a básica.

A condutividade manteve-se bem próxima dos resultados apresentados por BUENO et al. (2008) para o mesmo reservatório. Quando esse parâmetro é elevado, pode subentender-se que há um indicador de poluição, o que não foi encontrado para este estudo.

A temperatura da água e o oxigênio dissolvido não variaram com a profundidade. Como há circulação de água por entre o braço do reservatório, a temperatura não oscila bruscamente e está diretamente ligada ao perfil do oxigênio.

PÁDUA et al. (1997) estudaram a variação diurna de parâmetros limnológicos em viveiros de piscicultura e relatam que as variáveis mantiveram-se bem próximas em todos os horários e profundidades. Como um viveiro de piscicultura tem profundidade relativamente baixa, quando comparada a reservatórios, e como o trabalho de PÁDUA (1997) foi realizado durante o dia, houve menor variação dos parâmetros físicos e químicos. Em regiões tropicais, lagos rasos podem ter estratificação e desestratificação térmica no período de 24 horas. Contudo, os ambientes estudados apresentam dinâmicas totalmente diferentes. SIPAÚBA-TAVARES \& DURIGAN (1995), pesquisando as variações dos parâmetros abióticos em regime semiintensivo de criação de peixes, destacam a importância da entrada e saída de água, que, se associadas com as variáveis limnológicas, contribuem ou prejudicam uma produção. GOLOMBIESKI et al. (2005) ressaltam que o policultivo de peixes em consórcio com cultura de arroz não acarreta problemas limnológicos, ficando as variáveis, de modo geral, dentro das condições de conforto para a espécie e da faixa estabelecida pela legislação.

O nitrito, a amônia e o fósforo total apresentaram diferenças significativas entre os estratos. O nitrito teve maior concentração no hipolímnio, diferenciando-se estatisticamente $(p<0,05)$ do epilímnio e do metalímnio. Segundo ESTEVES (1998), em lagos oligotróficos, durante todo o ano não se observa estratificação do nitrito. Já em ambientes eutróficos, essa situação é percebida no hipolímnio. Neste trabalho, o valor do 
nitrito apresentado é baixo, e essa concentração, conforme a Resolução do CONAMA 357/05, não interfere na produção de peixes em tanques-rede em reservatórios, ficando abaixo do limite permitido. Entretanto, cerca de $70 \%$ da carga anual de fósforo e nitrogênio que chegam aos corpos d'água são procedentes de fontes não pontuais como atividades agrícolas (OLIVEIRA et al., 2008).

A amônia teve distribuição parecida com o nitrato, sendo que no hipolímnio apresentou diferenças significativas $(\mathrm{p}<0,05)$ quando comparado ao epilímnio. Segundo ESTEVES (1998), o sedimento é o principal sítio de realização do processo de formação de amônia. Ainda assim, essa não obteve teores maiores que o permitido pela Resolução do CONAMA 357/05.

$\mathrm{O}$ fósforo total obteve níveis muito maiores na parte inferior do reservatório $(\mathrm{p}<0,05)$, porém, não afeta negativamente na produção de organismos aquáticos. Esse valor pode, também, estar relacionado com o lançamento de rações na água, sendo que a parte não aproveitada pelos peixes acaba se depositando no fundo, fazendo com que ocorra a elevação desse mineral, principalmente em situações de falta de oxigênio. Como no presente estudo pressupõe-se que há circulação total de água, o que pode influenciar essa concentração são as fontes exógenas, como os alimentos ofertados aos peixes. BUENO et al. (2008) encontraram valores de fósforo, para o mesmo reservatório, em torno de 28,41 IET, índice de eutrofização considerado baixo. Os autores relatam também que o fósforo no sedimento diminui com o passar do tempo. Como no presente trabalho as coletas não foram feitas ao longo do tempo, não houve períodos de chuva para que houvesse remoção dessa concentração, deixando os minerais sedimentados.

MACEDO \& SIPAÚBA-TAVARES (2005), estudando o estado trófico em ambientes sequenciais de criação de peixes, encontraram aumento no grau de trofia do primeiro em direção ao último viveiro, o que pode estar ligado, dentre outras coisas, à velocidade e renovação da água. BACCARIN et al. (2000), em avaliação de fontes de fósforo e nitrogênio nos tanques de cultivo de tilápias-vermelhas, mostraram que a qualidade de água não se deteriora, ficando dentro das condições adequadas de cultivo e também da legislação. Segundo MENEZES \& BEYRUTH (2003), o tipo de cultivo que se desenvolve, ao se utilizar tanques-rede, propõe maior concentração de matéria orgânica, embora não promova a deterioração da qualidade da água. ALVES \& LEONARDO (2005) relatam que a atividade de criação de tilápias em tanques-rede causa alterações no ecossistema aquático. SAMPAIO \& BRAGA (2005) e SANTOS et al. (2008) informam resultados opostos, em que os parâmetros físicos e químicos da água no cultivo de tilápias em tanques-rede mantiveram-se dentro do limite imposto pela legislação 357/05 do CONAMA, não causando implicações significativas no ambiente aquático.

Muita atenção se tem dado ao conforto das espécies nos locais de produção e pouca sobre a qualidade da água nesses ambientes. O aporte exagerado de ingredientes alimentares e as densidades elevadas de estocagem podem acarretar a eutrofização do meio onde os peixes são criados, condição essa possível de afetar a capacidade de suporte, resultando em problemas na produção. Portanto, práticas de manejo que favoreçam o desempenho zootécnico dos animais e não influenciem negativamente no ecossistema aquático, além do constante acompanhamento anual das variáveis físicas e químicas da água destas áreas, são necessárias para o sucesso da atividade.

\section{CONCLUSÕES}

Ocorreu variação nictimeral, exceto para o fósforo. Entretanto as variáveis estão dentro dos limites recomendados para a aquicultura, com exceção do oxigênio dissolvido, que apresentou valores baixos. Para a distribuição vertical, as concentrações dos parâmetros físicos e químicos da água não ultrapassaram o limite estabelecido pela Resolução do CONAMA 357/05 para criação de peixes. No entanto, houve variação vertical para nitrito.

\section{REFERÊNCIAS}

ALVES, R. C. P.; LEONARDO, A. E. B. Caracterização do impacto da tilapicultura em tanques-rede sobre os parâmetros físicoquímicos da água. In: REUNIÃO CIENTÍFICA DA CAUNESP, 2005, Jaboticabal, SP. Anais... Jaboticabal, 2005.

APHA. Standard methods for the examination of water and wastewater. 20. ed. Washington: American Public Health Associoation, $1998.824 \mathrm{p}$. 
ARANA, L. V. Princípios químicos da qualidade de água em aqüicultura: uma revisão para peixes e camarões. 2. ed. Florianópolis, Editora da UFSC, 2004. 231 p.

AYROZA, D. M. M. R.; FURLANETO, F. P. B.; AYROZA, L. M. $\mathrm{S}$. Regularização dos projetos de tanques-rede em águas públicas continentais de domínio da união no Estado de São Paulo. Boletim do Instituto de Pesca, n. 36, p. 1-32, 2006.

BACCARIN, A. E.; FRASCÁ-SCORVO, C. M. D.; NOVATO, P. F. C. Níveis de nitrogênio e fósforo na água de tanques de cultivo de tilápias vermelhas submetidas a diferentes manejos alimentares. Acta Scientiarum, v. 22, n. 2, p. 485-489, 2000.

BUENO, G. W.; MARENGONI, N. G.; JÚNIOR, A. C. G.; BOSCOLO, W. R.; TEIXEIRA, R. A. Estado trófico e bioacumulação do fósforo total no cultivo de peixes em tanques-rede na área aquícola do reservatório de Itaipu. Acta Scientiarum Biological Sciences, v. 30, n. 3, p. 237-243, 2008.

CARNEIRO, P. C. F.; CYRINO, J. E. P.; CASTAGNOLLI, N. Produção da tilápia vermelha da Flórida em tanques-rede. Scientia Agrícola, v. 56, n. 3, p. 673-679, 1999.

CHAGAS, E. C.; GOMES, L. C.; JUNIOR, H. M.; ROUBACH, R. Produtividade de tambaqui criado em tanque-rede com diferentes taxas de alimentação. Ciência Rural, v. 37, n. 4, p. 1109-1115, 2007.

CONAMA - Conselho Nacional do Meio Ambiente. Resolução 357 de 17 de março de 2005. Disponível em: <http://www.mma. gov.br/port/conama/res/res05/res35705.pdf $>$. Acesso em: 18 jun. 2009.

ESTEVES, F. A. Fundamentos de limnologia. 2. ed. Rio de Janeiro: Interciência, 1998.

GOLOMBIESKI, J. I.; MARCHEZAN, E.; MONTI, M. B.; STORCK, L.; CAMARGO, E. R.; SANTOS, F. M. Qualidade da água no consórcio de peixes com arroz irrigado. Ciência Rural, v. 35, n. 6, p. 1263-1268, 2005.

GRAEFF, A.; PRUNER, E. N. Variáveis que podem interferir na sobrevivência e desenvolvimento da tilápia nilótica (Oreochromis niloticus) na região fria do Estado de Santa Catarina. In: IV CONGRESSO IBEROAMERICANO VIRTUAL DE ACUICULTURA. COMUNICACIÓN CIENTÍFICA, 5., 2006, Zaragoza, Espanha. Anais... Zaragoza, Espanha, p. 70-79, 2006.

GUNKEL, G. Limnología de un lago tropical de Alta Montaña, en Ecuador: características de los sedimentos y tasa de sedimentación. Revista de Biología Tropical, v. 51, n. 2, p. 381-390, 2003.

HAKANSON, L.; PARPAROV, A.; HAMBRIGHT, K. D. Modelling the impact of water level fluctuations on water quality (suspended particulate matter) in Lake Kinneret, Israel. Ecological Modelling, v. 128, p. 101-125, 2002.

KOROLEFF. Determination of nutrients. In: GRASSHOFF, K. (Ed.). Methods of sea water analysis. Verlag Chemie Weinhein, 1976. p. 117-181.

KUBITZA, F. Qualidade da água na produção de peixes. Piracicaba: ESALQ, 1999. 51 p.

LIMA, C. B.; OLIVEIRA, E. G.; ARAÚJO FILHO, J. M.; SEIXAS SANTOS, F. J.; PEREIRA, W. E. Qualidade da água em canais de irrigação com cultivo intensivo de tilápia nilótica (Oreochromis niloticus). Revista Ciência Agronômica, v. 39, n. 4, p. 531-539, 2008 .

MACEDO, C. F.; SIPAÚBA-TAVARES, L. H. Variações de nutrientes e estado trófico em viveiros sequenciais de criação de peixes. Acta Scientiarum Animal Sciences, v. 27, n. 3, p. 405411, 2005.

MACKERETH, J. F. H.; HERON, J.; TALLING, J. F. Water analysis: some revised methods for limnologists. Freshwater Biological Association, n. 36, 1978.

MENEZES, L. C. B.; BEYRUTH, Z. Impactos da aquicultura em tanques-rede sobre a comunidade bentônica da represa de Guarapiranga, São Paulo, SP. Boletim do Instituto de Pesca, v. 29, n. 1, p. 77-86, 2003.

PADUA, D. M. C.; SIPAÚBA-TAVARES, L. H.; SILVA, P. C.; PADUA, J. T. Variação diurna de parâmetros limnológicos em viveiros de piscicultura. Anais da Escola Agronômica e Veterinária, v. 57, n. 1, p. 93-102, 1997.

RIBEIRO, G. M.; MAIA, C. E.; MEDEIROS, J. F. Uso da regressão linear para estimativa da relação entre a condutividade elétrica e a composição iônica da água de irrigação. Revista Brasileira de Engenharia Agrícola e Ambiental, v. 9, n.1, p. 15-22, 2005.

SALVADOR, R.; MÜLLER, E. E.; LEONHARDT, J. H.; PRETTO-GIORDANO, L. G.; DIAS, J. A.; FREITAS, J. C.; MORENO, A. M. Isolamento de Streptococcus spp. de tilápias do Nilo (Oreochromis niloticus) e qualidade da água de tanques rede na Região Norte do Estado do Paraná, Brasil. Semina, Ciências Agrárias, v. 24, n. 1, p. 35-42, 2003.

SAMPAIO, J. M. C.; BRAGA, L. G. T. Cultivo de tilápias em tanques-rede na Barragem do Ribeirão de Salmonéa, Floresta Azul, Bahia. Revista Brasileira de Saúde e Produção Animal, v. 6, n. 2, p. 42-52, 2005.

SANTOS, E. C.; FREITAS, S. H.; DIAS FILHO, V. A.; GUERRA, A. J. S. Avaliação limnológica nictimeral no cultivo de tilápia (Oreochromis niloticus) em tanque-rede. Disponível 
em: <http://ppg.revistas.uema.br/index.php/REPESCA/article/ viewFile/81/88>. Acesso em: 22 jun. 2009.

SAS Institute Inc. SAS User's guide statistics. 9. ed. Cary, North Caroline: SAS Institute Inc., 2004.

SILVA, A. P.; MENDES, P. P. Influência de duas dietas na qualidade da água dos tanques-berçário, utilizados no cultivo do camarão marinho Litopenaeus vannamei (Boone, 1931). Acta Scientiarum Animal Sciences, v. 28, n. 1, p. 105-111, 2006.

SIPAÚBA-TAVARES, L. H.; DURIGAN, J. G. Variação dos fatores abióticos e pigmentos totais em dois viveiros de criação de peixes em regime semi-intensivo. Acta Limnologica Brasiliensia, v. 7, p. 10-22, 1995.

STRICKLAND, J. D. H.; PARSONS, T.R. 1972. A pratical handbook of sea water analysis. Ottawa: Fish Res. Board of Canada, 1972. 310 p.

ZAR, J. H. Biostatistical analysis. $2^{\text {nd }}$ ed. New Jersey, USA: Prentice Hall, Englewood Cliffs, 1984. 718 p.

ZIMERMANN, S.; RIBEIRO, R. P.; VARGAS, L.; MOREIRA, H. L. M. Fundamentos da moderna aqüicultura.Canoas: Ed. ULBRA, 2001. 200 p.

Submetido em: 25 jul. 2009. Aceito em: 30 dez. 2009. 